\title{
A diffusion-consumption problem for oxygen in a living tissue perfused by capillaries
}

\section{Andro Mikelić}

LaPCS, UFR Mathématiques

Bât. Doyen Jean Braconnier

Université Claude Bernard Lyon 1

69622 Villeurbanne Cedex

FRANCE

e-mail: Andro.Mikelic@univ-lyon1.fr

Mario Primicerio

Dipartimento di Matematica " Ulisse Dini "

Viale Morgagni 67/A

I-50134 Firenze, ITALIA 


\begin{abstract}
We study a mathematical model describing the nonlinear diffusion of oxygen in a living tissue, in presence of consumption due to metabolism. The tissue is perfused by a system of parallel capillaries in which oxygen is carried by the blood in the form of gas freely diffusing in plasma and bound to hemoglobine. We prove global existence of a unique smooth solution to the resulting parabolichyperbolic system.
\end{abstract}




\section{Chapter 1}

\section{Introduction}

It is well known that oxygen is supplied to living tissues through microcirculation of blood. The first attempt to describe the phenomenon in mathematical terms is the classical Krogh's model (see [13], [14]). This model deals with an idealized geometrical arrangement consisting of one capillary of circular cross section concentric with a circular cross section of muscle tissue; the exchange of oxygen is modeled through a law of Robin's type (flux proportional to the jump between partial pressure of $\mathrm{O}_{2}$ in blood and in the tissue) and a diffusion problem in axial symmetry with a consumption term has to be solved in the region occupied by the tissue.

Many extensions of Krogh's model have been proposed and studied (see [6], [20] for a survey) and several semianalytical or approximated methods have been developed, also to incorporate the effect of the presence of many capillaries.

The number of papers devoted to this subject in last 2-3 decades is really impressive and we will just quote a few of them, referring the interested reader to the literature quoted therein.

We single out three main lines of research: (i) modeling the mechanism of transport/storage of $\mathrm{O}_{2}$ in microcirculation and of transport/storage/consumption of oxygen in living tissue ([10], [18], [20]); (ii) discussing the boundary conditions that express the exchange across the walls of capillaries ([5], [7], [19], [21]), and (iii) finding approximated solutions often based on the use of line sources 
to mimic the presence of capillaries and/or on asymptotic expansions ([1], [4], [5], [11], [22]).

Our approach is based on the discussion carried out on topics (i) and (ii) and has the aim of obtaining a rigorous mathematical result on the well-posedness (existence, uniqueness, dependence on the data) of the corresponding analytical problem.

In [16] we already considered the problem of perfusion of living tissue by a bundle of parallel capillaries and we discussed the corresponding homogenization.

In the present paper we release some assumptions that were instrumental for the proofs of [16]: the fact that the transport of $O_{2}$ in blood was supposed to be based only on convection and the assumption of instantaneous equilibrium between oxygen in plasma and bound to erythrocytes.

Thus, the model we deal with is more similar to the one presented in [17]. The analysis given there is heavily based on symmetry (just one capillary surrounded by a co-axial cylindrical slab of tissue as in the original Krogh's model, while in our case we have $N$ capillaries of radii $\left.R_{i}, i=1,2 \ldots N\right)$, and on the use of classical representation techniques that are clearly inapplicable to our general geometric situation. Our existence and uniqueness results could be compared with the study of smooth solutions for two-scale quasilinear parabolic systems, arising in modeling of catalytic reactors, in [8].

The plan of the paper is the following.

In Section 2 we give the mathematical formulation of the problem, just recalling the basic physiological facts (see [12] for a comprehensive introduction to mathematical physiology), and we prove a simple a-priori estimate; in Section 3 we consider several auxiliary problems which are necessary in the proof of the existence theorem which is given in Section 4 and is based on Schauder's fixed point theorem. The solution is sufficiently smooth, as it will be clear by the arguments. In the last Section we prove the uniqueness. 


\section{Chapter 2}

\section{Formulation of the problem and basic assumptions}

Let $O$ be a bounded set in $\mathbf{R}^{2}$ with smooth boundary. Let $\mathbf{x} \equiv(x, y, z)$ and denote by $\Omega \equiv\{\mathbf{x} \equiv(x, y, z):(x, y) \in O, 0<z<L\}$. For $i=1,2, \ldots, N$ denote by $\mathcal{C}_{i} \equiv\left\{\mathbf{x}:\left(x-x_{i}\right)^{2}+\left(y-y_{i}\right)^{2}<R_{i}{ }^{2}, 0<z<L\right\}$, and by $\mathcal{C}=\bigcup_{i=1}^{N} \mathcal{C}_{i}$.

We will assume that the living tissue occupies $\omega=\Omega \backslash \mathcal{C}$, and represent the bundle of capillaries. We assume that $\partial \mathcal{C}$ and $\partial \Omega$ have no common parts outside $z=0$ and $z=L$.

We have to find $2 N+1$ functions: $C(\mathbf{x}, t)$, defined in $\omega \times \mathbf{R}^{+}$, represents the volumetric concentration of oxygen diffusing in the tissue; $c_{i}(z, t)$ and $\ell_{i}(z, t)$, defined on $\mathcal{C}_{i} \times \mathbf{R}^{+}(i=1,2 \ldots N)$, represent the concentrations of oxygen in the blood flowing in the $i$-th capillary, respectively dissolved in plasma and bound to hemoglobin.

Oxygen diffuses in the tissue according to mass balance equation:

$$
\frac{\partial \mathcal{M}(C)}{\partial t}-D \triangle C=Q(C)
$$

where $D$ is the diffusion coefficient, $Q \leq 0$ represents, in absolute value, the rate of oxygen consumption, and $\mathcal{M}(C)$ is the total oxygen content of a unit volume of tissue, that is a monotone function of the concentration $C$ of freely diffusing oxygen. To be specific, we can think of a law of type Michaelis-Menten

$$
\mathcal{M}(C)=C+\lambda C^{p}\left(C^{p}+k^{p}\right)^{-1}
$$


where $\lambda$ and $k$ are positive constants and a typical value for $p$ is 2.5 (see [12], [19]). Assuming (2.2) corresponds to postulate that the mass of oxygen contained in the unit volume of the tissue is the sum of the mass $C$ of the freely diffusing oxygen(e.g. to myoglobin) which is assumed to be in instantaneous equilibrium with the former.

Of course, the equation (2.1) can also include nonlinear diffusivity. Explicit dependence on $\mathbf{x}$ and $t$ will be excluded to avoid additional technical complications.

At the boundaries $\partial \mathcal{C}_{i}$, i.e. at the walls of capillaries, we assume that oxygen flow is induced by deviations from the osmotic equilibrium (Henri's law) and we write

$$
C_{i}(\theta, z, t)-\nu c_{i}(z, t)=\beta \frac{\partial C_{i}}{\partial r}, i=1 \ldots N,
$$

where $\nu$ and $\beta$ are positive constants and we denoted by $C_{i}(\theta, z, t)$ the value of $C$ at point $\left(x_{i}+R_{i} \cos \theta, y_{i}+R_{i} \sin \theta, z\right)$ and at time $t$ (where $\theta$ is an angular coordinate) and by $\frac{\partial C_{i}}{\partial r}$ its derivative, normal to $\partial C_{i}$ and pointing toward tissue, at the same point and time. ${ }^{1}$

Mass balance of oxygen in each $\mathcal{C}_{i}$ will include convection (with given speed $u(t)$ of the blood, say in the positive $z$ direction) for both $\ell_{i}$ and $c_{i}$, diffusion in axial direction for $c_{i}$, and exchange with the surrounding tissue. If $\alpha$ and $1-\alpha$ represent the volume fraction of the blood occupied by plasma and erythrocytes, respectively, and $d$ is the diffusivity of oxygen in plasma, we will write

$$
\begin{aligned}
& \alpha\left\{\frac{\partial c_{i}}{\partial t}+u(t) \frac{\partial c_{i}}{\partial z}-d \frac{\partial^{2} c_{i}}{\partial z^{2}}\right\}+(1-\alpha)\left\{\frac{\partial \ell_{i}}{\partial t}+u(t) \frac{\partial \ell_{i}}{\partial z}\right\}= \\
& =\frac{D}{\pi R_{i}{ }^{2}} \int_{0}^{2 \pi} \frac{\partial C_{i}}{\partial r} R_{i} d \theta, i=1 \ldots N .
\end{aligned}
$$

From now on, we will assume that $u(t)$ is a given positive $C^{1}$-function. Finally, we will have to postulate a relationship between $\ell_{i}$ and $c_{i}$. In [16] we assumed a law of instantaneous equilibrium $\ell_{i}=\gamma\left(c_{i}\right), \gamma$ being a monotone increasing

\footnotetext{
${ }^{1} \mathrm{~A}$ generalization of (2.3) in which the normal derivative $\frac{\partial C_{i}}{\partial r}$ is a monotone function of $C_{i}-\nu c_{i}$ could be also treated with only minor changes.
} 
function with $\gamma_{i}(0)=0$. Here, we make the more general assumption that a relaxation mechanism toward equilibrium is given: so that a positive constant $\tau$ and a monotone function $\Phi(\Phi(0)=0)$ exist, so that:

$$
\tau\left\{\frac{\partial \ell_{i}}{\partial t}+u(t) \frac{\partial \ell_{i}}{\partial t}\right\}=\Phi\left(\gamma\left(c_{i}\right)-\ell_{i}\right), i=1 \ldots N .
$$

The problem is completed by prescribing the following conditions:

(i) initial conditions:

$$
\begin{cases}C(\mathbf{x}, 0)=C^{0}(\mathbf{x}), & \mathbf{x} \in \omega, \\ c_{i}(z, 0)=c_{i}^{0}(z), & z \in(0, L), i=1 \ldots N \\ \ell_{i}(z, 0)=\ell_{i}^{0}(z), & z \in(0, L), i=1 \ldots N\end{cases}
$$

(ii) inlet/outlet boundary conditions for the capillaries:

$$
\begin{cases}c_{i}(0, t)=c_{i 0}(t), & t>0, i=1 \ldots N \\ c_{i}(L, t)=c_{i L}(t), & t>0, i=1 \ldots N, \\ \ell_{i}(0, t)=\ell_{i 0}(t), & t>0, i=1 \ldots N\end{cases}
$$

(iii) boundary conditions for the tissue, that we will take simply as homogeneous Neumann conditions:

$$
\frac{\partial C}{\partial n}=0, \text { on } \partial \Omega \backslash \partial \mathcal{C}, t>0
$$

We note that, incorporating $\alpha$ and $1-\alpha$ in the definition of $c_{i}$ and $\ell_{i}$ and renormalizing variables, the problem reduces to the following, where the same symbols have been used to save notation:

$$
\begin{aligned}
& \frac{\partial \mathcal{M}(C)}{\partial t}-\triangle C=Q(C) \text { in } \omega \times \mathbf{R}^{+} \\
& C_{i}(\theta, z, t)-c_{i}(z, t)=\beta \frac{\partial C_{i}(\theta, z, t)}{\partial r} \text { on } \partial \mathcal{C}_{i} \times \mathbf{R}^{+} \\
& \frac{\partial c_{i}}{\partial t}+u \frac{\partial c_{i}}{\partial z}-d \frac{\partial^{2} c_{i}}{\partial z^{2}}+\frac{\partial \ell_{i}}{\partial t}+u \frac{\partial \ell_{i}}{\partial z}=\frac{K}{\pi R_{i}} \int_{0}^{2 \pi} \frac{\partial C_{i}}{\partial r} d \theta, \text { in } \mathcal{C}_{i} \times \mathbf{R}^{+} \\
& \frac{\partial \ell_{i}}{\partial t}+u \frac{\partial \ell_{i}}{\partial z}=\Phi\left(\gamma\left(c_{i}\right)-\ell_{i}\right), \text { in } \mathcal{C}_{i} \times \mathbf{R}^{+}
\end{aligned}
$$


where $i=1,2 \ldots N$ in (2.10)-(2.12) and the initial and boundary conditions are given by (2.6)-(2.8). ${ }^{2}$

We make the following assumptions on functions $\mathcal{M}, Q, \Phi, \gamma$ appearing in (2.9)-(2.12).

(H1) $\mathcal{M}$ is a positive strictly increasing locally Lipschitz continuous function defined on $[0,+\infty)$.

(H2) $Q$ is a non-positive locally Lipschitz continuous function defined on $[0,+\infty)$, $Q(0)=0$.

(H3) $\Phi$ is an increasing locally Lipschitz continuous function defined on $(-\infty,+\infty)$, $\Phi(0)=0, \Phi^{\prime}(0)>0$.

(H4) $\gamma$ is a strictly increasing locally Lipschitz continuous function defined on $[0,+\infty), \gamma(0)=0$.

Concerning the notations and the functional spaces, we follow the reference [15]

We prove the following a-priori estimate.

Proposition 2.1. Assume that initial and boundary data for $c_{i}$ and $C$ are strictly positive and smaller than a constant E. Then, if

$$
E_{1}=\gamma(E)
$$

and if data for $\ell_{i}$ are strictly positive and less than $E_{1}$, any classical solution of problem (2.6)-(2.12) is such that

$$
\begin{aligned}
& 0<C(\mathbf{x}, t)<E, \quad \mathbf{x} \in \omega, t>0 \\
& 0<c_{i}(z, t)<E, \quad z \in[0, L], t>0, i=1 \ldots N \\
& 0<\ell_{i}(z, t)<E_{1}, \quad z \in[0, L], t>0, i=1 \ldots N .
\end{aligned}
$$

\footnotetext{
${ }^{2}$ We could allow positive quantities $\beta$ and $K$ to depend on $i$.
} 
Proof. We confine ourselves to prove the upper bound. If the second inequality is violated in any of (2.14)-(2.16), then a $t_{0}>0$ should exist such that for $t<t_{0}$ they hold and one of the following cases occur:

(a) $\ell_{i}\left(z_{0}, t_{0}\right)=E_{1} \quad$ for some $z_{0}$ and $i$, while $C\left(\mathbf{x}, t_{0}\right)<E, \mathbf{x} \in \omega$ and $c_{i}\left(z, t_{0}\right)<E$ in $(0, L) \forall i$;

(b) $c_{i}\left(z_{0}, t_{0}\right)=E \quad$ for some $z_{0}$ and $i$, while $C\left(\mathbf{x}, t_{0}\right)<E, \mathbf{x} \in \omega$ and $\ell_{i}\left(z, t_{0}\right) \leq E_{1}$ in $(0, L) \forall i$.

(c) $C\left(\mathbf{x}_{\mathbf{0}}, t_{0}\right)=E$ for some $\mathbf{x}_{\mathbf{0}}$ and $\ell_{i}\left(z, t_{0}\right) \leq E_{1}, c_{i}\left(z, t_{0}\right) \leq E, z \in(0, L), \forall i$.

In case (a), first we note that $z_{0} \neq 0, z_{0} \neq L$ so that $\ell_{i z}\left(z_{0}, t_{0}\right)=0$. Hence (2.12) implies

$$
\Phi\left(\gamma\left(c_{i}\left(z_{0}, t_{0}\right)\right)-E_{1}\right) \geq 0
$$

but since $c_{i}<E, \gamma\left(c_{i}\right)<E_{1}$ and (2.17) contradicts (H3).

In case (b), again $z_{0} \neq 0, z_{0} \neq L$, so that $c_{i z}=0, c_{i z z} \leq 0, c_{i t} \geq 0$. Moreover, from (2.12):

$$
\ell_{i t}+u \ell_{\left.i z\right|_{z_{o}, t_{o}}}=\Phi\left(\gamma(E)-\ell_{i}\right) \geq 0 .
$$

Consequently, from (2.11) $\int_{0}^{2 \pi} C_{\left.i r\right|_{z_{o}, t_{o}}} d \theta \geq 0$, which is in contradiction with (2.10) and (b).

Finally, maximum principle applied to (2.9) ensures that if $C$ attains a maximum value in $\omega \times\left(0, t_{0}\right]$, it should be attained at some point of $\partial \mathcal{C}_{i} \times\left\{t_{0}\right\}$ where $C_{i r}$ has to be strictly negative, according to boundary point principle. But this contradicts $(2.10)$.

Corollary 2.2. Let (H1)-(H4) and the assumptions of Proposition 2.1 hold. Then functions $\mathcal{M}, Q, \gamma, \Phi$ in (2.9), (2.12) can be truncated, i.e. there is no loss of generality in assuming that

(H5) Functions $\mathcal{M},|Q|,|\Phi|, \gamma$ are Lipschitz continuous and bounded by a constant $\hat{M}$. 


\section{Chapter 3}

\section{Preliminary results}

Denote by $S_{T}=\{(z, t): 0<z<L, 0<t<T\}$ and consider the following

Problem A. For any given non-negative $w \in H^{\alpha, \alpha / 2}\left(S_{T}\right) \cap C\left(\bar{S}_{T}\right)$, find $\ell(z, t) \in$ $C\left(\bar{S}_{T}\right)^{1}$ and satisfying

$$
\begin{aligned}
& \ell_{t}+u(t) \ell_{z}=\Phi(\gamma(w)-\ell) \quad \text { in } S_{T}, \\
& \ell(z, 0)=\ell^{0}(z) \geq 0, \quad 0<z<L, \\
& \ell(0, t)=\ell_{0}(t) \geq 0, \quad 0<t<T .
\end{aligned}
$$

We prove

Proposition 3.1. Let the chain of data (3.2), (3.3) be continuous ${ }^{2}$, and denote by $\ell_{0}$ MAX its maximum. Then Problem A has a unique solution; moreover

$$
0 \leq \ell \leq \gamma(W), \quad \text { in } S_{T},
$$

where

$$
W=\max \left(\|w\|_{C\left(S_{T}\right)}, \gamma^{-1}\left(\ell_{0 M A X}\right)\right)
$$

Furthermore, if the chain of data is Hölder continuous with the exponent $\alpha \in$ $(0,1)$, the solution is Hölder continuous with exponent $\alpha / 2$.

\footnotetext{
${ }^{1}$ In fact its material derivative corresponding to the velocity $u(t) \vec{e}_{z}$ is also continuous.

${ }^{2}$ As usual, by this expression we mean that data themselves are continuous and zero-order compatibility conditions are satisfied (in this case $\ell^{0}(0)=\ell_{0}(0)$ ).
} 
Proof. To find $\ell(z, t)$ it is sufficient to integrate an ordinary differential equation along the characteristics $z-\int_{0}^{t} u(\tau) d \tau=$ const. Hence $\ell(z, t)$ is continuously differentiable along the tangential direction to the characteristics. The normal direction enters as a parameter and inherits the smoothness of the chain of data. Estimate (3.4) follows at once using assumptions (H3) and (H4).

Next, we want to investigate how the solution $\ell[w]$ of Problem $A$ depends on $w$ and we prove

Proposition 3.2. Under the same assumptions, there exists a constant $k>0$ such that

$$
\begin{aligned}
& \left\|\ell\left[w^{\prime}\right]-\ell\left[w^{\prime \prime}\right]\right\|_{L^{\infty}\left(0, T ; L^{2}(0, L)\right)}+\sqrt{\min _{0 \leq t \leq T} u(t)}\left\|\ell\left[w^{\prime}\right]-\ell\left[w^{\prime \prime}\right]\right\|_{L^{2}\left(0, T ; L^{\infty}(0, L)\right)} \\
& \leq k\left\|w^{\prime}-w^{\prime \prime}\right\|_{L^{\infty}\left(0, T ; L^{2}(0, L)\right)} .
\end{aligned}
$$

Proof. For given $w^{\prime}$ and $w^{\prime \prime}$ find $\ell\left[w^{\prime}\right]$ and $\ell\left[w^{\prime \prime}\right]$ solving Problem $A$ and let $\bar{\ell}=\ell\left[w^{\prime}\right]-\ell\left[w^{\prime \prime}\right]$. Then $\bar{\ell}(z, t)$ solves

$$
\bar{\ell}_{t}+u(t) \bar{\ell}_{z}=\left[\Phi\left(\gamma\left(w^{\prime}\right)-\ell^{\prime}\right)-\Phi\left(\gamma\left(w^{\prime}\right)-\ell^{\prime \prime}\right)\right]+\left[\Phi\left(\gamma\left(w^{\prime}\right)-\ell^{\prime \prime}\right)-\Phi\left(\gamma\left(w^{\prime \prime}\right)-\ell^{\prime \prime}\right)\right]
$$

with zero initial and boundary data. Multiply (3.7) by $\bar{\ell}$ and note that the first term on the r.h.s. of the equation so obtained is negative, while the second term is dominated by $\Lambda^{2}\left|w^{\prime}-w^{\prime \prime}\right||\bar{\ell}|$, where $\Lambda$ is the largest of the Lipschitz constants of $\Phi$ and of $\gamma$. Integrating over $S_{T}$ concludes the proof.

Remark 3.3. The calculations as in Proposition 3.2 are justified by regularization. For such calculations in the the theory of the first order semi-linear hyperbolic equations see e.g. [2] . Using the technique from [2] it is straightforward to prove that for $\ell_{0} \in B V(0, T)$ and $\ell^{0} \in B V(0, L)$ we have $\ell \in L^{\infty}(0, T ; B V(0, L))$ and

$$
\|\ell\|_{L^{\infty}(0, T ; B V(0, L))} \leq C\left\{\left\|\ell_{0}\right\|_{B V(0, T)}+\left\|\ell^{0}\right\|_{B V(0, L)}+\left\|\partial_{z} w\right\|_{L^{1}((0, L) \times(0, T))}\right\}
$$

We note that this regularity doesn't require the compatibility of $\ell^{0}$ and $\ell_{0}$. 
Next, for $i=1,2 \ldots N$ we consider the following

Problem B. For any $w_{i}$ as in Problem $A$ and for any given positive constant $\lambda_{i}$ and any non-negative function $A_{i} \in H^{\beta, \beta / 2}\left(S_{T}\right) \cap C\left(\bar{S}_{T}\right)$ find $c_{i} \in W_{q}{ }^{2,1}\left(S_{T}\right) \cap$ $C\left(\bar{S}_{T}\right)(\forall q \in[2,+\infty))$ such that

$$
\begin{aligned}
& c_{i t}+u(t) c_{i z}-d c_{i z z}=-\Phi\left(\gamma\left(c_{i}\right)-\ell\left[w_{i}\right]\right)+A_{i}(z, t)-\lambda_{i} c_{i}, \text { in } S_{T}, \\
& c_{i}(z, 0)=c_{i}{ }^{0}(z) \geq 0, \quad 0<z<L, \\
& c_{i}(0, t)=c_{i 0}(t) \geq 0, \quad 0<t<T, \\
& c_{i}(L, t)=c_{i L}(t) \geq 0, \quad 0<t<T .
\end{aligned}
$$

From now on we drop index $i$ for simplicity. We prove

Proposition 3.4. Let the chain of the data (3.10)-(3.12) be Hölder continuous and denote by $c_{0}$ MAX its maximum. Then Problem B has a unique solution; moreover

$$
0 \leq c \leq M \quad \text { in } S_{T}
$$

where

$$
M=\max \left(c_{0 M A X}, W,\|A\|_{C\left(S_{T}\right)} / \lambda\right) .
$$

Proof. ¿From Theorem 6.4 and Theorem 6.7 of [15] chapter V it follows that Problem $B$ has a solution such that

$$
c \in \stackrel{0}{V}_{2,2}^{1,0}\left(S_{T}\right) \cap H^{\alpha, \alpha / 2}\left(\bar{S}_{T}\right), \quad \alpha>0,
$$

where

$$
\begin{aligned}
& V_{2,2}^{1,0} \equiv\left\{\varphi \in L^{\infty}\left(0, T ; L^{2}(0, L)\right): \varphi_{z} \in L^{2}\left(S_{T}\right),\right. \\
&\left.\int_{0}^{T} h^{-2}\|\varphi(z, t+h)-\varphi(z, t)\|_{L^{2}\left(S_{T-h}\right)}^{2} d h<+\infty\right\} .
\end{aligned}
$$

Moreover, since $c \in W_{q}^{2,1}\left(S_{T}\right)$ for any $q>1$ (see [15], Chapter 4, and recall boundedness of $\Phi$ and $A$ ) we have

$$
c_{z} \in H^{\lambda, \lambda / 2}\left(S_{T}\right), \quad \forall \lambda<1 .
$$


To prove uniqueness, denote by $c^{\prime}$ and $c^{\prime \prime}$ two possible solutions and let

$$
\bar{c}(z, t)=c^{\prime}(z, t)-c^{\prime \prime}(z, t) .
$$

We have

$$
\bar{c}_{t}+u \bar{c}_{z}-d \bar{c}_{z z}+\lambda \bar{c}=\Phi\left(\gamma\left(c^{\prime \prime}\right)-\ell[w]\right)-\Phi\left(\gamma\left(c^{\prime}\right)-\ell[w]\right)
$$

with $\bar{c}=0$ on the parabolic boundary of $S_{T}$. Multiply (3.18) by $\bar{c}$ and integrate over $S_{T}$. Using (H3) and (H4) we conclude that $\bar{c} \equiv 0$. Next, use maximum principle noting that the r.h.s. of (3.9) is non-negative for $c=0$ and non-positive for $c=M$.

It is clear that, for fixed $A$ and $\lambda$, solving Problem $A$ for any $w$ and then Problem $B$ for $\ell[w]$ defines a mapping

$$
c=c[w]
$$

According to Proposition (3.1) and (3.4), if

$$
|w| \leq \max \left\{\gamma^{-1}\left(\ell_{0 \mathrm{MAX}}\right), c_{0 \mathrm{MAX}},\|A\|_{C\left(S_{T}\right)} / \lambda\right\} \equiv \bar{M}
$$

then

$$
|c[w]| \leq \bar{M}
$$

Hence $c[w]$ maps the ball with radius $\bar{M}$ of $C\left(S_{T}\right)$ into itself.

Moreover, since

$$
\left\|c_{z}\right\|_{H^{\lambda, \lambda / 2}} \leq k
$$

where $k$ only depends on $\bar{M}$, the mapping is compact. To prove that the mapping is continuous we take a sequence $\left\{w^{m}\right\}$ and study the corresponding $c^{m}=c\left[w^{m}\right]$. Since

$$
\left\|c_{z}^{m}\right\|_{H^{\lambda, \lambda / 2}\left(S_{T}\right)}+\left\|c^{m}\right\|_{W_{q}^{2,1}} \leq k
$$


there is a subsequence $c^{r}$ converging to $\hat{c}(z, t)$ uniformly and converging weakly in $W_{q}^{2,1}$. Hence, passing to the limit (along the subsequence) in the equation satisfied by $c\left[w^{r}\right]$ we obtain that $c\left[w^{r}\right] \rightarrow c[w]$. Using Schauder fixed point theorem gives us the existence theorem for the following

Problem C. For any given $\lambda_{i}>0$ and non-negative $A_{i} \in H^{\beta, \beta / 2}\left(S_{T}\right) \cap C\left(\bar{S}_{t}\right)$ solve Problem $B$ with $\ell\left[w_{i}\right]$ replaced by $\ell\left[c_{i}\right]$.

Now (dropping again index $i$ ) we investigate the dependence of $c$ upon $A$ and prove

Proposition 3.5. Let $c^{\prime}$ and $c^{\prime \prime}$ be solutions of Problem C corresponding to $A^{\prime}$ anf $A^{\prime \prime}$ respectively. Then, for any $t \in(0, T)$ it is:

$$
\int_{0}^{L}\left|c^{\prime}(z, t)-c^{\prime \prime}(z, t)\right| d z \leq k\left\|A^{\prime}-A^{\prime \prime}\right\|_{L^{1}\left(S_{T}\right)} .
$$

Proof. Writing again $\bar{c}=c^{\prime}-c^{\prime \prime}$, it is

$$
\bar{c}_{z}+u(t) \bar{c}_{z}-d \bar{c}_{z z}+\lambda \bar{c}=\Phi\left(\gamma\left(c^{\prime \prime}\right)-\ell\left[c^{\prime \prime}\right]\right)-\left(\Phi\left(\gamma\left(c^{\prime}\right)-\ell\left[c^{\prime}\right]\right)+A^{\prime}-A^{\prime \prime}\right.
$$

Testing (3.25) with regularized $\operatorname{sign}(\bar{c})$ we get

$$
\int_{o}^{L}|\bar{c}(z, t)| d z+\lambda \iint_{S_{t}}|\bar{c}| d z d \tau+\iint_{S_{t}}\left(\Phi^{\prime \prime}-\Phi^{\prime}\right) \operatorname{sign} \bar{c} \leq \iint_{S_{T}}\left|A^{\prime}-A^{\prime \prime}\right| d z d \tau
$$

(the meaning of $\Phi^{\prime}$ and $\Phi^{\prime \prime}$ is obvious).

Now, proceeding as in the proof of Proposition 3.2, the integral containing $\Phi^{\prime \prime}$ $\Phi^{\prime}$ is estimated in terms of $\iint_{S_{t}}\left|\ell\left[c^{\prime \prime}\right]-\ell\left[c^{\prime}\right]\right| d z d \tau$. Then using Proposition (3.2) the proof is concluded.

Therefore we have

Corollary 3.6. Solution to Problem C is unique.

Now, we state and solve our last auxiliary problem.

Problem D. For any given $n$-tuple $\underline{\varphi} \equiv\left\{\varphi_{1} \ldots \varphi_{N}\right\}$ of non-negative functions $\varphi_{i} \in L^{\infty}\left(S_{T}\right)$ find $C \in L^{\infty}\left(Q_{T}\right) \cap V_{2}^{1,1 / 2}\left(Q_{T}\right)\left(Q_{T}=\omega \times(0, T)\right)$, such that

$$
\frac{\partial}{\partial t} \mathcal{M}(C)-\Delta C=Q(C) \text { in } Q_{T}
$$




$$
\begin{aligned}
& C(\mathbf{x}, 0)=C^{0}(\mathbf{x}), \quad \mathbf{x} \in \omega, \\
& \frac{\partial C}{\partial n}=0, \quad \text { on } \partial \Omega \backslash \partial \mathcal{C} \times(0, T), \\
& {\left[C-\beta C_{r}\right]_{i}=\varphi_{i}, \quad \text { on } \partial \mathcal{C}_{i} \times(0, T),}
\end{aligned}
$$

where we wrote for simplicity $\left[C-\beta C_{r}\right]_{i}$ to indicate that the quantity in bracket has to be evaluated for $x=x_{i}+R_{i} \cos \theta, y=y_{i}+r_{i} \sin \theta$.

We prove

Proposition 3.7. If $C^{0} \in L^{\infty}(\omega)$ is a given non-negative function, then Problem $\mathrm{D}$ is uniquely solvable in $Q_{T}$. Moreover

$$
0 \leq C \leq \max \left\{\max C^{0}, \max _{i}\left\|\varphi_{i}\right\|_{L^{\infty}\left(S_{T}\right)}\right\}
$$

and there exist positive constants $\delta, k_{1}$ and $k_{2}$ such that

$$
\|C\|_{V_{2}^{1,1 / 2}\left(Q_{T}\right)}+\|C\|_{H^{\delta, \delta / 2}\left(\bar{Q}_{T}\right)} \leq k_{1} \sum_{i}\left\|\varphi_{i}\right\|_{L^{\infty}\left(S_{T}\right)}+k_{2}
$$

where

$$
\|C\|_{V_{2}^{1,1 / 2}\left(Q_{T}\right)}=\|\nabla C\|_{L^{2}\left(Q_{T}\right)}^{2}+\int_{0}^{T-h} d t \int_{\omega} h^{-1}|C(\mathbf{x}, t+h)-C(\mathbf{x}, t)|^{2} d x .
$$

Proof. Using the classical theory of linear parabolic equations with discontinuous coefficients from [15], together with the Schauder fixed point theorem, we arrive at solvability of the Problem $D$. The membership of $C$ in $V_{2}^{1,1 / 2}\left(Q_{T}\right)$ and estimate (3.32) follow from classical theory of parabolic equations.

Uniqueness is obtained by the theory of entropy solutions (see [3]). It can also be seen that constants $k_{1}$ and $k_{2}$ exist such that

$$
\|C\|_{H^{\delta, \delta / 2}\left(\bar{Q}_{T}\right)} \leq k_{1} \sum_{i}\left\|\varphi_{i}\right\|_{L^{\infty}\left(S_{T}\right)}+k_{2}
$$

For more details we refer to [15], pages 418-423. From maximum principle and assumptions (H1), (H2), it is immediately seen that $C \geq 0$.

Moreover, since $Q$ is non-positive, and $\beta>0$ the upper bound for $C$ is obtained at once. 
Corollary 3.8. Let $C^{0}(\mathbf{x}) \in C^{2}(\bar{\omega})$ and that its normal derivative vanishes on $\partial \omega \backslash \mathcal{C}$; moreover let $\varphi \in C\left[\bar{S}_{T}\right], \varphi_{i z} \in H^{\beta, \beta / 2}$ be chosen so that (3.30) holds initially. Then the problem (3.27)-(3.30) has a unique solution $C \in$ $H^{2+\beta, 1+\beta / 2}\left(Q_{T}\right)$.

Proof. From Theorem 7.4 page 491 of [15] we find that under our assumptions a solution of Problem $D$ exists in the class specified.

Remark 3.9. Having stated the problem in a class of very smooth functions, we require lot of smoothness on the data. Some generalizations are however possible. 


\section{Chapter 4}

\section{Existence theorem}

Denote by $K(M, T)$ the set of all $n$-tuple of functions $\varphi_{i} \in C\left(\bar{S}_{T}\right)$ and such that

$$
0 \leq \varphi_{i}(z, t) \leq M, \quad i=1, \ldots N, z \in S_{T}
$$

Solve Problem $D$ with this choice of $\varphi \equiv\left\{c_{1}, c_{2} \ldots c_{N}\right\}$ and let

$$
A_{i}(z, t)=\frac{\lambda_{i}}{2 \pi} \int_{0}^{2 \pi} C\left(x_{i}+R_{i} \cos \theta, y_{i}+R_{i} \sin \theta, z, t\right) d \theta, \quad i=1,2, \ldots N .
$$

Now, for each $i$, solve Problem $C$ and find an $n$-tuple $\mathbf{c} \equiv\left\{c_{1}, \ldots, c_{N}\right\}$.

Thus, we have defined a mapping

$$
\mathbf{c}=\mathcal{T}[\varphi],
$$

and we have

Proposition 4.1. Let $\lambda_{i}=\frac{2 K}{\beta R_{i}}$. If $\underline{\hat{c}}$ is a fixed point of mapping (4.3)

$$
\underline{\hat{c}}=\mathcal{T}[\hat{c}],
$$

then our problem is solved by the $2 N+1$ functions

$$
\hat{\boldsymbol{c}}, \ell[\hat{\boldsymbol{c}}], C[\hat{\boldsymbol{c}}]
$$

where $\boldsymbol{\ell}[\hat{\boldsymbol{c}}] \equiv\left\{\ell_{1}\left[\hat{c}_{1}\right], \ell_{2}\left[\hat{c}_{2}\right], \ldots, \ell_{N}\left[\hat{c}_{N}\right]\right\}$ is obtained solving Problem A and $C[\hat{\boldsymbol{c}}]$ is obtained solving Problem D.

In order to prove that the mapping $\mathcal{T}$ has a fixed point we should establish its properties. First we prove 
Proposition 4.2. There exists $M>0$ such that

$$
\varphi \in K_{M, T} \Rightarrow \mathcal{T}[\varphi] \in K_{M, T}
$$

for any given $T>0$.

Proof. Take

$$
M>\max \left\{\left\|C^{0}\right\|_{C(\bar{\omega})}, c_{0 M A X}, \gamma^{-1}\left(\ell_{0 M A X}\right)\right\}
$$

and recall (3.4), (3.13), (3.21), (3.31).

Now we prove

Proposition 4.3. $\mathcal{T}$ maps $K_{M, T}$ into a compact subset.

Proof. Proposition 3.7 guarantees that (3.31) and (3.32) are satisfied. This ensures that $C[\boldsymbol{c}]$ belongs to a set $\mathcal{A}$ which is compact in $L^{2}\left(0, T ; H^{\alpha}(\omega)\right)$ for any $\alpha<1$. Then $\mathcal{T}[\boldsymbol{c}]$ is uniformly bounded in $W_{q}^{2,1}$ (recall (3.23)) and hence compact in $K_{M, T}$ for $q>3$.

Proposition 4.4. $\mathcal{T}$ is continuous.

Proof. Let $\mathbf{c}_{m} \rightarrow \mathbf{c}$ in $K_{M, T}$. Then the set $\mathcal{A}$ is compact in $L^{2}\left(0, T, H^{\alpha}(\omega)\right)$ for any $\alpha<1$ as seen above and $C\left[\boldsymbol{c}_{m}\right]$ contains a subsequence converging strongly in $L^{2}\left(0, T ; H^{\alpha}(\omega)\right)$, weakly in $V_{2}^{1,1 / 2}\left(Q_{T}\right)$ and weak* in $L^{\infty}\left(Q_{T}\right)$ to a solution $C$ of Problem D. Because of the uniqueness, the whole sequence converges.

Moreover, from Proposition 3.2 we have that $\boldsymbol{\ell}\left[\mathbf{c}_{m}\right]$ converges in $L^{1}\left(S_{T}\right)$ and $\mathcal{T}\left[\mathbf{c}_{n}\right]$ are uniformly bounded in $W_{q}^{2,1}\left(S_{T}\right)$ so that they converge uniformly and weakly in $W_{q}^{2,1}$.

Finally, w $=\lim \mathcal{T}\left[\boldsymbol{c}_{n}\right]$ satisfies Problem $C$ and, because of uniqueness, the whole sequence converges.

Hence we have proved the following result 
Corollary 4.5. Let us suppose hypothesis (H1)-(H5). Let $C^{0}$ be a non-negative bounded function. Let $c^{0} \in C^{2}[0, L]$ and $c_{0}, c_{L} \in C^{1}[0, T]$ be non-negative vector valued functions satisfying zero-order compatibility condition. Let $\ell^{0}$ and $\ell_{0}$ be non-negative vector functions of bounded variation. Then there is $M>0$ such that the mapping $\mathcal{T}$ has at least one fixed point $\hat{c} \in K_{M, T}$. Furthermore, $\left\{\hat{\boldsymbol{c}}, \boldsymbol{\ell}[\hat{\boldsymbol{c}}], C[\hat{\boldsymbol{c}}\} \in W_{q}^{2,1}\left(\mathcal{C}_{i} \times(0, T)\right)^{N} \times L^{\infty}\left(0, T ; B V\left(\mathcal{C}_{i}\right)\right)^{N} \times V_{2}^{1,1 / 2}(\omega \times(0, T))\right.$, $\forall q \geq 2$.

Supposing a bit more of regularity, we find that solution is very regular :

Theorem 4.6. Let the assumptions of Corollary 4.5 be satisfied and let in addition $C^{0} \in C^{3}(\bar{\omega})$ and let chain of data (3.2)-(3.3) be Hölder continuous, with exponent $\lambda \in(0,1)$. Furthermore, let the compatibility condition (3.30) be satisfied at $t=0$. Then the problem (2.6)-(2.12) has a non-negative solution $\left\{\hat{\boldsymbol{c}}, \boldsymbol{\ell}[\hat{\boldsymbol{c}}], C[\hat{\boldsymbol{c}}\} \in W_{q}^{2,1}\left(\mathcal{C}_{i} \times(0, T)\right)^{N} \times H^{\lambda}\left(\overline{\mathcal{C}}_{i} \times[0, T]\right)^{N} \times H^{2+\beta, 1+\beta / 2}(\omega \times(0, T))\right.$ , $\forall q \geq 2$ and $\beta \in(0,1)$.

Remark 4.7. Let in addition $c^{0} \in C^{3}[0, L]$ and $c_{0}, c_{L} \in C^{2}[0, T]$ be non-negative vector valued functions satisfying zero and first-order compatibility condition. Then $\hat{\boldsymbol{c}} \in H^{2+\lambda, 1+\lambda / 2}\left(\mathcal{C}_{i} \times(0, T)\right)^{N}$. 


\section{Chapter 5}

\section{Uniqueness Theorem}

The uniqueness theorem is somehow unexpected, except with very high regularity. We note that even for a special case of our model, studied in [17], no uniqueness result was obtained. In fact for the uniqueness we don't really need classical solutions. The regularity $V_{2}^{1,1 / 2}(\omega \times(0, T)) \times W_{q}^{2,1}\left(\mathcal{C}_{i} \times(0, T)\right)^{N} \times$ $L^{\infty}\left(0, T ; B V\left(\mathcal{C}_{i}\right)\right)^{N}$ is enough, but we have to balance carefully the corresponding " energy "terms. It should be noted that the presence of $\mathcal{M}$ makes the calculations with the time derivatives and time differences tricky. Only optimal arrangement of the terms from 3 equations gives the right conclusion.

Theorem 5.1. The problem (2.6)-(2.12) has a unique bounded non-negative solution $\{C, c, \ell\} \in V_{2}^{1,1 / 2}(\omega \times(0, T)) \times W_{q}^{2,1}\left(\mathcal{C}_{i} \times(0, T)\right)^{N} \times L^{\infty}\left(0, T ; B V\left(\mathcal{C}_{i}\right)\right)^{N}, q>$ 3.

Proof. Let us suppose that there exist two solutions for the problem (2.6)(2.12). Then the difference of the solutions, denoted by $\{C, c, \ell\}$, is once more in $V_{2}^{1,1 / 2}(\omega \times(0, T)) \times W_{q}^{2,1}\left(\mathcal{C}_{i} \times(0, T)\right)^{N} \times L^{\infty}\left(0, T ; B V\left(\mathcal{C}_{i}\right)\right)^{N}$. We note that there are $N$ capillary tubes $\mathcal{C}_{i}$ of the length $L$ and consequently functions $c$ and $\ell$ are vector valued with $N$ components.

We proceed in several steps.

\section{STEP}

We integrate the equation (2.9) in time and get

$\mathcal{M}\left(C_{1}(x, t)\right)-\mathcal{M}\left(C_{2}(x, t)\right)-D \Delta \int_{0}^{t} C(x, \xi) d \xi=\int_{0}^{t}\left(Q\left(C_{1}\right)-Q\left(C_{2}\right)\right) d \xi$ 
Consequently for every $\varphi \in H^{1}(\omega)$ we have

$$
\begin{aligned}
& \int_{\omega}\left(\mathcal{M}\left(C_{1}(x, t)\right)-\mathcal{M}\left(C_{2}(x, t)\right)\right) \varphi d x+D \int_{\omega} \nabla\left(\int_{0}^{t} C\right) \nabla \varphi d x+ \\
& \left.\left.\frac{D}{\beta} \sum_{i} \int_{0}^{L} \int_{0}^{2 \pi} R_{i} \varphi\right|_{r=R_{i}} \int_{0}^{t} C\right|_{r=R_{i}} d \vartheta d z=\int_{\omega}\left(\int_{0}^{t}\left(Q\left(C_{1}\right)-Q\left(C_{2}\right)\right) \varphi d \xi\right) d x \\
& +\left.\left.\frac{D}{\beta} \sum_{i} \int_{0}^{L} \int_{0}^{2 \pi} R_{i} \varphi\right|_{r=R_{i}} \int_{0}^{t} c\right|_{r=R_{i}} d \vartheta d z
\end{aligned}
$$

We take $\varphi=C$ as a test function and get

$$
\begin{aligned}
& \int_{0}^{t} \int_{\omega}\left(\mathcal{M}\left(C_{1}(x, \xi)\right)-\mathcal{M}\left(C_{2}(x, \xi)\right)\right) C d x d \xi+\frac{D}{2} \int_{\omega}\left|\nabla\left(\int_{0}^{t} C\right)\right|^{2} d x+ \\
& \left.\frac{D}{2 \beta} \sum_{i} \int_{0}^{L} \int_{0}^{2 \pi} R_{i}\left(\int_{0}^{t} C\right)^{2}\right|_{r=R_{i}} d \vartheta d z=\int_{0}^{t} \int_{\omega}\left(\int_{0}^{\xi}\left(Q\left(C_{1}\right)-Q\left(C_{2}\right)\right) C d \xi d x\right. \\
& +\left.\left.\frac{D}{\beta} \sum_{i} \int_{0}^{t} \int_{0}^{L} \int_{0}^{2 \pi} R_{i} C\right|_{r=R_{i}} \int_{0}^{t} c\right|_{r=R_{i}} d \vartheta d z
\end{aligned}
$$

Since

$$
\mid \int_{0}^{t} \int_{\omega}\left(\int_{0}^{\xi}\left(Q\left(C_{1}\right)-Q\left(C_{2}\right)\right) C d \xi d x \mid \leq C_{q} t \int_{0}^{t} \int_{\omega} C^{2}\right.
$$

and

$$
\begin{aligned}
& \left.\left|\frac{D}{\beta} \sum_{i} \int_{0}^{t} \int_{0}^{L} \int_{0}^{2 \pi} R_{i} C\right|_{r=R_{i}} \int_{0}^{t} c\right|_{r=R_{i}} d \vartheta d z \mid= \\
& \mid \frac{D}{\beta} \sum_{i} \int_{0}^{L} \int_{0}^{2 \pi} R_{i}\left(\left.\left.\int_{0}^{t} C\right|_{r=R_{i}} \int_{0}^{t} c\right|_{r=R_{i}}-\int_{0}^{t}\left(\left.\left.c\right|_{r=R_{i}} \int_{0}^{\xi} C\right|_{r=R_{i}}\right) d \xi d \vartheta d z \mid \leq\right. \\
& \frac{D^{2}}{\beta} \sum_{i} \int_{0}^{L} \int_{0}^{2 \pi} R_{i}\left(\left.\int_{0}^{t} c^{2}\right|_{r=R_{i}}+\left(\left.\int_{0}^{t} c\right|_{r=R_{i}}\right)^{2}\right)+ \\
& \frac{D}{4 \beta} \sum_{i} \int_{0}^{L} \int_{0}^{2 \pi} R_{i}\left(\left.\left(\int_{0}^{t} C\right)^{2}\right|_{r=R_{i}}+\left(\int_{0}^{t}\left(\left.\int_{0}^{\xi} C\right|_{r=R_{i}}\right)^{2} d \xi\right)\right)
\end{aligned}
$$

Hence for $t \leq T_{0}=\min \left\{T, c_{m} /\left(2 c_{q}\right)\right\}$ we have

$$
\begin{aligned}
& \int_{\omega}\left|\nabla\left(\int_{0}^{t} C\right)\right|^{2} d x+\int_{0}^{t} \int_{\omega} C^{2}+\left.\sum_{i} \int_{0}^{L} \int_{0}^{2 \pi} R_{i}\left(\int_{0}^{t} C\right)^{2}\right|_{r=R_{i}} \\
& \leq\left. C_{M} \sum_{i} \int_{0}^{t} \int_{0}^{L} \int_{0}^{2 \pi} R_{i} c^{2}\right|_{r=R_{i}}
\end{aligned}
$$


Now we study the equation for $\ell$ :

$$
\frac{\partial \ell}{\partial t}+u(t) \frac{\partial \ell}{\partial z}=\left(\Phi\left(\gamma\left(c_{1}\right)-\ell_{1}\right)-\Phi\left(\gamma\left(c_{2}\right)-\ell_{2}\right)\right) \quad \text { in }(0, L) \times(0, T)
$$

By testing this equation by regularized $\ell$ and after using Proposition 3.2, we get

$$
\int_{0}^{t} \int_{0}^{L} \ell^{2} \leq C_{\rho} t \int_{0}^{t} \int_{0}^{L} c^{2} \quad \text { for every i }
$$

\section{STEP}

Finally we study the equation for $c$ :

$$
\begin{aligned}
& \frac{\partial c}{\partial t}+u(t) \frac{\partial c}{\partial z}-d \frac{\partial^{2} c}{\partial z^{2}}+\frac{2 D}{\beta R_{i}} c=-\left(\Phi\left(\gamma\left(c_{1}\right)-\ell_{1}\right)-\Phi\left(\gamma\left(c_{2}\right)-\ell_{2}\right)\right) \\
& +\left.\frac{D}{\beta \pi R_{i}} \int_{0}^{2 \pi} C\right|_{r=R_{i}} d \vartheta \quad \text { in }(0, L) \times(0, T)
\end{aligned}
$$

We integrate (5.9) from 0 to $t$ and test the obtained equation by $c$. Then we have

$$
\begin{aligned}
& \int_{0}^{L} c^{2}(t)+\int_{0}^{L} c(t)\left(\int_{0}^{t} u(\eta) \frac{\partial c}{\partial z} d \eta\right)+\frac{d}{2} \partial_{t} \int_{0}^{L}\left|\frac{\partial}{\partial z} \int_{0}^{t} c\right|^{2}+\frac{2 D}{\beta R_{i}} \partial_{t} \int_{0}^{L}\left(\int_{0}^{t} c\right)^{2} \leq \\
& C_{0}\left(\int_{0}^{L} \int_{0}^{2 \pi}\left|\int_{0}^{t} C\right||c|+\int_{0}^{L}|c| \int_{0}^{t}|c|+\int_{0}^{L}|c| \int_{0}^{t}|\ell|\right)
\end{aligned}
$$

Clearly, we should first take care of the transport term :

$$
\begin{aligned}
& \left|\int_{0}^{t} \int_{0}^{L} c(\xi)\left(\int_{0}^{\xi} u(\eta) \frac{\partial c}{\partial z} d \eta\right) d x d \xi\right|=\mid \int_{0}^{t} \int_{0}^{L}\left(\partial_{\xi}\left(\int_{0}^{\xi} u(\eta) \frac{\partial c}{\partial z} d \eta \int_{0}^{\xi} c(\eta) d \eta\right)\right. \\
& \left.-u(\xi) \frac{\partial c}{\partial z}(\xi) \int_{0}^{\xi} c\right) d x d \xi \mid \leq C_{t r}\left(\frac{1}{\delta} \int_{0}^{t} \int_{0}^{L}\left|\frac{\partial}{\partial z} \int_{0}^{t} c\right|^{2}+\right. \\
& \left.\left(\delta+\frac{t\|u\|_{\infty}}{d}\right) \int_{0}^{t} \int_{0}^{L} c^{2}+\int_{0}^{L}\left|\frac{\partial}{\partial z} \int_{0}^{t} c\right|^{2}\right)
\end{aligned}
$$

After inserting (5.11) into (5.10) we get

$$
\begin{aligned}
& \sum_{i}\left(1-3 C_{0} \delta-C_{1} t\right) \int_{0}^{t} \int_{0}^{L} c^{2}(t)+\frac{d}{8} \int_{0}^{L}\left|\frac{\partial}{\partial z} \int_{0}^{t} c\right|^{2}+\frac{2 D}{\beta R_{i}} \int_{0}^{L}\left(\int_{0}^{t} c\right)^{2} \leq \\
& C_{3}\left(\left.\frac{1}{\delta} \int_{0}^{t} \int_{0}^{L} \int_{0}^{2 \pi} \sum_{i}\left|\int_{0}^{\xi} C\right|_{r=R_{i}}\right|^{2}+\frac{1}{\delta} \int_{0}^{t} \int_{0}^{L}\left|\frac{\partial}{\partial z} \int_{0}^{t} c\right|^{2}\right)
\end{aligned}
$$


Using (5.6) and Gronwall's inequality, we conclude that for $t \leq T_{1} \leq T_{0}$ and such that $1-3 C_{0} \delta-C_{1} T_{1}>0, c(x, t)=0$. Hence $C$ and $\ell$ are also zero.

Therefore we have uniqueness on a small time interval with length independent of the data. By repeating this procedure a finite number of times, we get uniqueness on $(0, T)$. 


\section{Bibliography}

[1] C. Bos, L. Hoof, T. Oostendorp, Mathematical model of erytrocytes as point-like sources, Math. Biosci. 125 (1995), 165-189.

[2] C. Bourdarias, Sur un système d'E.D.P. modélisant un processus d'adsorption isotherme d'un mélange gazeux, $\mathrm{M}^{2} \mathrm{AN}$ Mathematical Modelling and Numerical Analysis, Vol. 26 (1992), p. 867-892.

[3] J. Carrillo, Entropy Solutions for Nonlinear Degenerate Problems, Arch. Rational Mech. Anal., 147 (1999), 263-361.

[4] A. Dutta, A.S. Popel, A Theoretical analysis of intracellular oxyden diffusion, J. Theoret. Biol. 176 (1995), 165-174.

[5] C. D. Eggleton, A. Vardapalli, T. K. Roy, A. S. Popel, Calculation of intracapillary oxygen tension distribution in muscle, Math.Biosci., 167 (2000), 123-143.

[6] J.E. Fletcher, Mathematical modelling of the microcirculation, Math. Biosci. 38 (1978), 155-202.

[7] J.E. Fletcher, R.W. Shubert, On the computation of substrate levels in perfused tissues, Math. Biosci. 62 (1982), 75-106.

[8] A. Friedman, A. Tzavaras, A Quasilinear Parabolic System Arising in Modelling of Catalytic Reactors, J. of Differential Equations, 70 (1987), p. 167196. 
[9] J. Gonzalez - Fernandez, S. Atta, Transport and consumption of oxygen in capillary-tissue structure, Math. Biosci. 2 (1968), 225-261.

[10] L. Hoofd, Calculation of oxygen pressures in tissue with anisotropic capillary orientation, Math. Biosci. 129 (1995), 1-23.

[11] R. Hsu, T.W. Secomb, A Green's function method for analysis of oxygen delivery to tissue by microvascular networks, Math. Biosci. 96 (1989), 6178.

[12] J. Keener, J. Sneyd, Mathematical Physiology, Interdisciplinar Appl. Math. 8 Springer Verlag, 1998.

[13] A. Krogh, The number and distribution of capillaries in muscles with calculations of the oxygen pressure head necessary for supplying the tissue, J. Physiol. (London) 52 (1919), 409-415.

[14] A. Krogh, The Anatomy and Phisiology of Capillaries, Yale Univ. Press, New Haven 1929.

[15] O.A. Ladyženskaya, V.A. Solonnikov, N.N. Ural'ceva, Linear and QuasiLinear Equations of Parabolic Type, Translations of Mathematical Monographs Vol. 23, American Mathematical Society, Providence, 1968.

[16] A. Mikelić, M. Primicerio, Oxygen exchange between multiple capillaries and living tissues: An homogenization study, Rend. Mat. Acc. Lincei, Vol. 13 (2002), p. 149-164.

[17] C. V. Pao, Stability analysis of a coupled diffusion-transport system for oxygen transport in blood and tissue, Nonl. Anal. Th. Met. App. 9 (1985), 1037-1059.

[18] A. S. Popel, Analysis of capillary-tissue diffusion multicapillary system, Math. Biosci. 35 (1978), 187-211. 
[19] A.S. Popel, Mathematical modelling of oxygen transport near a tissue surface: effect of the surface $P_{O_{2}}$, Math. Biosci. 55 (1981), 231-246.

[20] A.S. Popel, Theory of oxygen transport to tissue, Crit. Riv. Biomed. Engrs., 17 (1989), 257-321.

[21] E.P. Salathé, T.C. Wang, J.F. Gross, Mathematical analysis of oxygen transport to tissue, Math. Biosci. 51 (1980), 89-115.

[22] M.S. Titicombe, M. J. Ward, An asymptotic study of oxygen transport from multiple capillaries to skeletral muscle tissue, SIAM J. Appl. Math., 60 (2000), 1767-1788. 